Research article

\title{
Guaranteed cost consensus protocol design for linear multi-agent systems with sampled-data information: An input delay approach
}

\author{
Yadong Zhao ${ }^{\mathrm{a}, \mathrm{b}}$, Weidong Zhang ${ }^{\mathrm{a}, \mathrm{b}, *}$ \\ a Department of Automation, Shanghai Jiao Tong University, Shanghai 200240, PR China \\ ${ }^{\mathrm{b}}$ Key Laboratory of System Control and Information Processing, Ministry of Education, Shanghai 200240, PR China
}

\section{A R T I C L E I N F O}

\section{Article history:}

Received 16 January 2016

Received in revised form

29 September 2016

Accepted 9 December 2016

\section{Keywords:}

Multi-agent systems

Consensus control

Sampled-data control

Guaranteed cost control

Linear matrix inequality

\begin{abstract}
A B S T R A C T
To investigate the energy consumption involved in a sampled-data consensus process, the problem of guaranteed cost consensus for sampled-data linear multi-agent systems is considered. By using an input delay approach, an equivalent system is constructed to convert the guaranteed cost consensus problem to a guaranteed cost stabilization problem. A sufficient condition for guaranteed cost consensus is given in terms of linear matrix inequalities (LMIs), based on a refined time-dependent Lyapunov functional analysis. Reduced-order protocol design methodologies are proposed, with further discussions on determining sub-optimal protocol gain and enlarging allowable sampling interval bound made as a complement. Simulation results illustrate the effectiveness of the theoretical results.
\end{abstract}

(c) 2016 ISA. Published by Elsevier Ltd. All rights reserved.

\section{Introduction}

In the past decade, the cooperative control of multi-agent systems has attracted compelling attention due to its widespread applications in spacecraft, vehicle formations, robotic teams and sensor networks [1-4], etc. The consensus problem, which requires all the agents reach an agreement on certain interest by using local relative information only, has been defined and formulated as a cooperative control problem in [5,6].

Most early studies regarding the consensus problem address the simple case where the agents are often restricted to be single, double or high-order integrators, with extensive consensus conditions established in $[3,7,8]$. To consider a more general case, consensus of multi-agent systems with linear time invariant (LTI) node dynamics were investigated in $[9,10]$, where the notion consensus region was introduced into protocol design. The results were then extended to synchronization of Lipschitz-type nonlinear dynamical networks in [11]. Instead of focusing on more complex node dynamics, remarkable efforts have been made to improve the performance of a consensus process. For instance, to achieve finite-time consensus in the absence or in the presence of external disturbances, carefully designed consensus algorithms were

\footnotetext{
* Corresponding author at: Department of Automation, Shanghai Jiao Tong University, Shanghai 200240, PR China.

E-mail addresses: ydzhao@outlook.com (Y. Zhao), wdzhang@sjtu.edu.cn (W. Zhang).
}

proposed in $[12,13]$ based on finite-time backstepping control technique. For more details about consensus design of continuoustime multi-agent systems, the survey paper [14] and references therein are recommended.

Note that most of the aforementioned literature on continuoustime multi-agent systems assume that the relative information is available for neighboring agents continuously, since the designed controllers are in a continuous-time structure. However, persistent interactions or remote sensing may be interrupted due to the unreliability of communication channels and the limitations of sensing abilities of agents. Hence, it is more practical to consider intermittent information transmission. Based on the above observation, sampled-data setting was introduced in [15-17], for second-order multi-agent systems under both fixed and timevarying topologies. By using zero-order holders for discretization, sufficient consensus conditions for sampled-data multi-agent systems were given in [11,18-21].

Though the communication and sensing loads can be reduced under the sampled-data setting, the control input energy and the state constraints are not considered. It motivates us to consider sampled-data implementation and guaranteed cost simultaneously for the consensus process. Fortunately, the guaranteed cost consensus problem for multi-agent systems with continuoustime information setting has been formulated and investigated recently, by taking a consensus LQR cost function. Sufficient guaranteed cost consensus conditions are given in [22-24]. Moreover, guaranteed cost consensus seeking for multi-sensor

http://dx.doi.org/10.1016/j.isatra.2016.12.003

0019-0578/৫ 2016 ISA. Published by Elsevier Ltd. All rights reserved.

Please cite this article as: Zhao Y, Zhang W. Guaranteed cost consensus protocol design for linear multi-agent systems with sampleddata information: An input delay approach. ISA Transactions (2016), http://dx.doi.org/10.1016/j.isatra.2016.12.003 
networks with Markov switching topologies was investigated in [25], with the motivation to reduce the energy consumption during consensus process. These meaningful works help us a lot in formulating the guaranteed cost consensus problem for multiagent systems with sampled-data information.

It should be noted that the majority of existing literature on sampled-data multi-agent consensus and guaranteed cost consensus focus on the case where the agents are governed by integrator-type dynamics. In this study, we consider the guaranteed cost consensus protocol design problem for sampled-data multiagent systems with general linear node dynamics and directed graphs. To the best of the authors' knowledge, there are few research results considering sampled-data implementation and guaranteed cost consensus simultaneously. To solve this problem, a reduced-order equivalent system is constructed to convert the guaranteed cost consensus problem to a guaranteed cost stabilization problem. A refined time-dependent Lyapunov functional [26] is then adopted to conduct the guaranteed cost analysis for the equivalent system. Moreover, guaranteed cost consensus condition and protocol design methods are established in terms of LMIs with the computational burden taken into account. The main contributions of the paper can be summarized as follows: (i) the concept of guaranteed cost is introduced into sampled-data multiagent systems by considering general linear node dynamics and directed graphs. (ii) A reduced-order equivalent system is constructed to convert the guaranteed cost consensus problem to the guaranteed cost stabilization problem. (iii) A refined sampled-data guaranteed cost consensus condition is given, which can be used to enlarge the allowable sampling interval bound for the sampling instants. (iv) A system decomposition approach is proposed for undirected graph case, so that the protocol can be synthesized via a numerically efficient LMI design.

The rest of the paper is organized as follows. In Section 2, we give some preliminaries on graph theory and LMI facts. In Section 3 , we formulate the guaranteed cost consensus problem for sampled-data multi-agent systems. We then present our main results concerning guaranteed cost consensus condition and protocol design methodology in Sections 4 and 5. Numerical studies are carried out in Section 6 to validate our design and a brief concluding remark is drawn in Section 7.

Notations: Throughout this paper, $\mathbb{R}$ denotes the field of real numbers. $\mathbb{R}_{+}$and $\mathbb{N}$ denote the set of positive real numbers and nonnegative integers, respectively. $\mathbb{R}^{n \times m}$ denotes the space of real $n \times m$ matrices. $I_{N}$ and $0_{N}$ represent, respectively, the identity matrix and the zero matrix of dimension $N . \mathbf{1}_{N}$ and $\mathbf{0}_{N}$ are defined as $\mathbf{1}_{N}=[1, \ldots, 1]^{T} \in \mathbb{R}^{N}$ and $\mathbf{0}_{N}=[0, \ldots, 0]^{T} \in \mathbb{R}^{N}$. $\|x\|$ denotes the 2 -norm of a vector $x$. $\operatorname{diag}\left\{A_{1}, \ldots, A_{n}\right\}$ represents a block-diagonal matrix with square matrices $A_{i}, i=1, \ldots, n$, on its diagonal. $\operatorname{col}\left\{a_{1}, a_{2}, \ldots, a_{N}\right\}$ denotes the column vector in the form of $\left[a_{1}^{T}, a_{2}^{T}, \ldots, a_{N}^{T}\right]^{T}$. The matrix inequality $A>B$ means that $A$ and $B$ are square Hermitian matrices, and $A-B$ is positive definite. $A \otimes B$ represents the Kronecker product of matrices $A$ and $B$. Matrices, if not explicitly stated, are assumed to have compatible dimensions.

\section{Preliminaries}

Let $\mathcal{G}=(\mathcal{V}, \mathcal{E})$ be a directed graph, where $\mathcal{V}=\left\{v_{1}, v_{2}, \ldots, v_{N}\right\}$ is a nonempty, finite set of nodes, and $\mathcal{E} \subseteq \mathcal{V} \times \mathcal{V}$ is the set of edges represented by ordered pairs of distinct nodes. A directed edge in $\mathcal{G}$ with parent node $v_{i}$ and child node $v_{j}$ is represented by $\left(v_{i}, v_{j}\right) \in \mathcal{E}$. Besides, a directed path of $\mathcal{G}$ from node $v_{s_{1}}$ to node $v_{s_{l}}$ is a sequence of edges of the form $\left(v_{s_{k}}, v_{s_{k+1}}\right), s_{k} \in 1, \ldots N, k=1, \ldots, l-1$. Undirected graphs are defined as special cases of directed graphs such that $\left(v_{i}, v_{j}\right) \in \mathcal{E}$ implies $\left(v_{j}, v_{i}\right) \in \mathcal{E}$, and an undirected path in $\mathcal{G}$ is defined analogously to a directed path.

The graph adjacency matrix $\mathcal{A}=\left[a_{i j}\right] \in \mathbb{R}^{N \times N}$ of $\mathcal{G}$ is defined such that $a_{i i}=0, a_{i j}>0$ if $\left(v_{j}, v_{i}\right) \in \mathcal{E}$, and $a_{i j}=0$ otherwise. Since $a_{i j}$ denotes the weight for the edge $\left(v_{j}, v_{i}\right)$, it can be assigned as 1 if $\left(v_{j}, v_{i}\right) \in \mathcal{E}$ and the corresponding weight are not relevant. If $\mathcal{G}$ is undirected, $a_{i j}$ is assumed to equal to $a_{j i}$ for $\forall i \neq j$. The Laplacian matrix $\mathcal{L}=\left[l_{i j}\right] \in \mathbb{R}^{N \times N}$ associated with $\mathcal{G}$ is defined as $l_{i i}=\sum_{j \neq i} a_{i j}$ and $l_{i j}=-a_{i j}, i \neq j$.

Definition 1 (Directed spanning tree). A directed graph $\mathcal{G}$ is said to have a directed spanning tree, if and only if $\mathcal{G}$ has at least one node with a directed path to all other nodes.

Remark 1. For an undirected graph $\mathcal{G}$, the existence of a directed spanning tree is equivalent to being connected. Thus the notion connected is used for the undirected graph $\mathcal{G}$.

Lemma 1 ([27]). Let $\mathcal{L} \in \mathbb{R}^{N \times N}$ be the Laplacian matrix of a directed graph $\mathcal{G}$, then the following statements hold: (i) $\mathcal{L}$ has at least one zero eigenvalue with $\mathbf{1}_{N}$ as the corresponding right eigenvector and all nonzero eigenvalues have positive real parts, (ii) zero is a simple eigenvalue of $\mathcal{L}$, and there exists a unique nonnegative left eigenvector $r$ of $\mathcal{L}$ associated with the zero eigenvalue, satisfying $r^{T} \mathcal{L}=0$ and $r^{T} \mathbf{1}_{N}=1$, if and only if $\mathcal{G}$ has a directed spanning tree.

Proposition 1 (Jensen's Inequality [26]). For any $n \times n$ matrix $R>0$, scalar $h>0$ and a vector function $\phi:[-h, 0] \rightarrow \mathbb{R}^{n}$ such that the integrations concerned are well-defined, the following holds:

$\int_{-h}^{0} \phi^{T}(s) R \phi(s) d s \geq \frac{1}{h} \int_{-h}^{0} \phi^{T}(s) d s R \int_{-h}^{0} \phi(s) d s$.

\section{Problem formulation}

We start by considering a multi-agent system consists of $N$ agents in the form

$\dot{x}_{i}(t)=A x_{i}(t)+B u_{i}(t), \quad i \in \mathcal{I}$,

where $x_{i} \in \mathbb{R}^{n}, \quad u_{i} \in \mathbb{R}^{m}$ are the state, input of agent $i$, and $\mathcal{I}=\{1, \ldots, N\}$. $A$ and $B$ are constant matrices with compatible sizes, and the pair $(A, B)$ is assumed to be stabilizable.

The information exchanges among these agents are modeled by digraphs. Let $v_{i}$ denote the $i$-th agent, and the communication graph among all the $N$ agents is defined such that $\mathcal{G}=(\mathcal{V}, \mathcal{E})$, where $\mathcal{V}=\left\{v_{1}, v_{2}, \ldots, v_{N}\right\}, \mathcal{E} \subseteq \mathcal{V} \times \mathcal{V}$. A directed edge $\left(v_{i}, v_{j}\right) \in \mathcal{E}$ implies that $v_{j}$ can obtain information from $v_{i}$, and $v_{i}$ is a neighbor of $v_{j}$. Self-loops in the form of $\left(v_{i}, v_{i}\right)$ are excluded from $\mathcal{E}$ when modeling. Let $\mathcal{N}_{i}$ denote the neighborhood index set of the $i$-th agent with $\mathcal{N}_{i}=\left\{j,\left(v_{j}, v_{i}\right) \in \mathcal{E}, i \neq j\right\}$.

In consensus design of linear multi-agent systems, linear relative-state feedback consensus law is widely used, due to its fundamentality and effectiveness. A general form of consensus laws of this type is considered in this paper, which is given as follows:

$u_{i}(t)=K \sum_{j \in \mathcal{N}_{i}}\left(x_{i}(t)-x_{j}(t)\right)$.

For the sake of simplicity, let $e_{i}$ denote the neighborhood synchronization error with respect to the $i$-th agent, that is, $e_{i}(t)=\sum_{j \in N_{i}}\left(x_{i}(t)-x_{j}(t)\right)$, then (3) can be written as,

$u_{i}(t)=K e_{i}(t)$.

To ensure that the above scheme solves the consensus problem for multi-agent systems, persistently remote sensing or interactions 
between adjacent agents are essential, since the protocol uses the neighborhood synchronization error instantly. Unfortunately, data losses and time-delays objectively exist in the sensor-controller links. To overcome these difficulties and reduce the sensing power, we turn to consider the following sampled-data setting, where the control signal is assumed to be generated by a zero-order hold $(\mathrm{ZOH})$ function,

$u_{i}(t)=K e_{i}\left(t_{k}\right), \quad t_{k} \leq t<t_{k+1}$,

with a sequence of hold times,

$0=t_{0}<t_{1}<\cdots<t_{k}<\cdots, \quad \lim _{k \rightarrow \infty} t_{k}=\infty$.

The sampling interval of sampling instants (6) can be either constant $t_{k+1}-t_{k}=h$ or variable with $k$-dependent $t_{k+1}-t_{k}=h_{k}$. The following assumption is imposed on the sampling interval.

Assumption 1. The sampling interval is bounded, i.e., $t_{k+1}-t_{k} \leq h, \forall k \in \mathbb{N}$, where $h$ is known.

Remark 2. Note that by using the protocol (5), the $i$-th agent only uses the error information between itself and its neighbors, meaning that it is in a distributed fashion. Moreover, all the agents in the team admit the same protocol structure and protocol parameters, which means that we only need to determine the protocol gain $K$ when implementing the consensus protocol.

Obviously, different choices of protocol gain $K$ and sampling instants (6) may result in different consensus performances. In this paper, we aim to use guaranteed cost consensus design to limit the consensus performance and determine the satisfactory protocol gain $K$. To achieve this goal, we need a cost function as performance index. By referring to [23], a reasonable choice of cost function is given as follows, which puts penalties on $e_{i}$ and $u_{i}$ :

$J=\sum_{i=1}^{N} \int_{t_{0}}^{\infty}\left\{e_{i}(t)^{T} Q e_{i}(t)+u_{i}^{T}(t) R u_{i}(t)\right\} d t$,

where $Q \in \mathbb{R}^{n \times n}$ and $R \in \mathbb{R}^{m \times m}$ are weighting matrices with $Q=Q^{T}>0$ and $R=R^{T}>0$. The cost function can be regarded an extension of interaction-related cost function introduced in [28] to general linear multi-agent systems. Moreover, the physical explanation of (7) for mobile sensor networks can be found in Remark 2 of [25].

Then the consensus problem and the guaranteed cost consensus problem for the sampled-data multi-agent systems are defined as follows.

Definition 2 (Consensus problem). Multi-agent system (2) with communication graph $\mathcal{G}$ is said to achieve consensus with sampled-data protocol (5) if there exists a protocol gain $K \in \mathbb{R}^{n \times m}$, such that all the agent states are driven to the same value, i.e., $\lim _{t \rightarrow \infty}\left\|x_{i}(t)-x_{j}(t)\right\|=0, \forall i, j \in I$.

Definition 3 (Guaranteed cost consensus problem). Multi-agent system (2) with communication graph $\mathcal{G}$ and cost function (7) is said to achieve guaranteed cost consensus with sampled-data protocol (5) if there exist a protocol gain $K \in \mathbb{R}^{n \times m}$ and $J^{*} \in \mathbb{R}_{+}$, such that the following two conditions hold for all sampling instants satisfying Assumption 1,

(i) The consensus problem is solved by protocol (5) with the protocol gain $K$.

(ii) The cost function (7) is upper-bounded by $J^{*}$, i.e., $J \leq J^{*}$.

Remark 3. By investigating the guaranteed cost consensus problem, the energy consumption involved in the consensus process is limited, and a tradeoff design between consensus performance and control effort of multi-agent systems can be achieved in the presence of variable sampling. The tradeoff design is realized by choosing appropriate weighting matrices. By choosing different $Q$, $R$ for a given $J^{*}$, the closed-loop multi-agent systems will exhibit different overall consensus performance, we refer the readers to the book [29] for more details about selecting $Q$ and $R$.

Before we move on to the next section, certain assumption should be imposed on $\mathcal{G}$.

Assumption 2. The communication graph $\mathcal{G}$ is directed and has a directed spanning tree.

Remark 4. We include the connected undirected graph case as a special case of Assumption 2 such that all edges in $\mathcal{G}$ are bidirectional.

\section{System transformation and guaranteed cost consensus condition}

The previous section defines the consensus problem and guaranteed cost consensus problem for multi-agent system (2) with sampled-data protocol (5). In this section, we aim to present some fundamental results concerning consensusability and guaranteed cost.

Motivated by the framework proposed by [9], which deals with the consensus problem of linear multi-agent system by system transformation and decomposition, we extend the results to sampled-data multi-agent systems and guaranteed cost consensus problems. It is worth mentioning that our extension is not direct as our method is based on singular value decomposition (SVD), and in our framework the dynamics and cost function are transformed simultaneously. Moreover, an input delay approach is applied, where the sampled-data multi-agent system is modeled as a continuous-time multi-agent system with delayed control input, for the convenience of guaranteed cost analysis.

To construct the equivalent system, we first represent the digital protocol (5) as a delayed input form, by letting $\tau(t)=t-t_{k}$, $t_{k} \leq t<t_{k+1}, \forall k \in \mathbb{N}$,

$\dot{x}_{i}(t)=A x_{i}(t)+B K \sum_{j=1}^{N} l_{i j} x_{j}(t-\tau(t))$.

Let $x(t)=\left[x_{1}^{T}(t), x_{2}^{T}(t), \ldots, x_{N}^{T}(t)\right]^{T} \in \mathbb{R}^{n N}$, it follows from (2) and (3) that,

$\dot{x}(t)=\left(I_{N} \otimes A\right) x(t)+(\mathcal{L} \otimes B K) x(t-\tau(t))$.

Let $r^{T}=\left[r_{1}, \ldots, r_{N}\right] \in \mathbb{R}^{1 \times N}$ be the left eigenvector of $\mathcal{L}$ associated with the simple zero eigenvalue satisfying $r^{T} \mathbf{1}_{N}=1$, and introduce the following disagreement variable for stability analysis:

$\delta(t)=\chi(t)-\left(\mathbf{1}_{N} r^{T} \otimes I_{n}\right) \chi(t)=\left(I_{N}-\mathbf{1}_{N} r^{T}\right) \chi(t)$.

From the proof of Theorem 1 in [9], we know that the consensus problem is solved if and only if $\lim _{t \rightarrow \infty}\|\delta(t)\|=0$. In fact, the consensus problem of the original sampled-data multi-agent system is converted as a stabilization problem of the following system:

$\dot{\delta}(t)=\left(I_{N} \otimes A\right) \delta(t)+(\mathcal{L} \otimes B K) \delta(t-\tau(t))$,

which is called as disagreement dynamics.

Apparently, in order to solve the guaranteed cost consensus problem, converting consensus to stabilization is not sufficient. The fact motivates us to consider converting guaranteed cost consensus to guaranteed cost stabilization. To this end, an extended framework which transforms the dynamics and the cost function simultaneously is considered. Since the cost function (7) is equivalent to 
$J=\int_{t_{0}}^{\infty}\left\{\delta^{T}(t)\left(\mathcal{L}^{T} \mathcal{L} \otimes Q\right) \delta(t)+\delta^{T}(t-\tau(t))\left(\mathcal{L}^{T} \mathcal{L} \otimes K^{T} R K\right) \delta(t-\tau(t))\right\} d t$,

where $\left(r^{T} \otimes I_{n}\right) \delta(t)=0_{n}, \quad \mathcal{L}^{T} \mathcal{L} \otimes Q \geq 0$ and $\mathcal{L}^{T} \mathcal{L} \otimes R \geq 0$, we construct a more compact equivalent system for the convenience of guaranteed cost consensus analysis by removing state equality constraint. The following matrices $W \in \mathbb{R}^{N \times(N-1)}$ and $Y \in \mathbb{R}^{N \times(N-1)}$ are defined for our analysis, such that the SVD of matrix $\mathcal{L}$ is:

$U=\left[W, \frac{r}{\|r\|}\right]^{T}, \quad V^{T}=\left[Y, \frac{1}{\sqrt{N}} \mathbf{1}_{\mathbf{N}}\right], \quad U \mathcal{L} V^{T}=\left[\begin{array}{cc}\Sigma & 0 \\ 0 & 0\end{array}\right]$,

where $\quad \Sigma=\operatorname{diag}\left\{\sigma_{N}, \sigma_{N-1}, \ldots, \sigma_{2}\right\} \in \mathbb{R}^{(N-1) \times(N-1)}, \quad \sigma_{N} \geq \sigma_{N-1} \geq \cdots \geq \sigma_{2}$ $>\sigma_{1}=0$.

Remark 5. From Assumption 2 and Lemma 1, we conclude that $\sigma_{N} \geq \sigma_{N-1} \geq \cdots \geq \sigma_{2}>\sigma_{1}=0$, since $\sigma_{i}=\sqrt{\lambda_{i} \lambda_{i}^{*}}, i \in \mathcal{I}$ and $\left\{0, \lambda_{2}, \ldots, \lambda_{N}\right\}$ is the spectrum of matrix $\mathcal{L}$ satisfying $0<\left|\lambda_{2}\right| \leq \cdots \leq\left|\lambda_{N}\right|$.

We then define a graph related matrix $\Xi$ for further analysis,

$\Xi \triangleq Y^{T} W \in \mathbb{R}^{(N-1) \times(N-1)}$

where $Y$ and $W$ are defined in (12). The matrix $\Xi$ has the following properties.

Lemma 2. Suppose that Assumption 2 holds, then $\Xi$ and $\Xi^{-1}$ are well-defined such that $\Xi^{-1}=W^{T}\left(I_{N}-\mathbf{1}_{N} r^{T}\right) Y$. Furthermore, if $\mathcal{G}$ is undirected, $\Xi=\Xi^{-1}=I$.

Proof. First, from Sylvester rank inequality and the fact that $\operatorname{rank}\left(Y^{T} W\right) \leq \min (\operatorname{rank}(Y), \operatorname{rank}(W))$, we know that $\operatorname{rank}(\Xi)=N-1$, which implies that $\Xi^{-1}$ exists. Next, since $U$ and $V$ are both orthogonal matrices, the following equations hold $W^{T} W=I_{N-1}, Y^{T} Y=I_{N-1}, W W^{T}=I_{N}-\frac{r r^{T}}{\|r\|^{2}}, Y Y^{T}=I_{N}-\frac{\mathbf{1}_{N} \mathbf{1}_{N}^{T}}{N}, W^{T} r=\mathbf{0}$, $Y^{T} \mathbf{1}_{N}=\mathbf{0}, \quad r^{T} \mathbf{1}_{N}=1$. Aa a result, $\Xi \cdot\left(W^{T}\left(I_{N}-\mathbf{1}_{N} r^{T}\right) Y\right)=I_{N-1}$ and $\Xi^{-1}=W^{T}\left(I_{N}-\mathbf{1}_{N} r^{T}\right) Y$. Furthermore, for the case that $\mathcal{G}$ is undirected, it is easy to check that $W=Y$ and $W^{T} Y=I_{N-1}$ according to $\sigma_{i}=\lambda_{i}>0$, which further imply that $\Xi=I_{N-1}=\Xi^{-1}$. Above all, we complete the proof.

Theorem 1. Suppose that Assumptions 1 and 2 hold, the distributed protocol (5) solves the consensus problem for multi-agent system (2) with communication graph $\mathcal{G}$, if and only if the following system:

$\dot{\xi}(t)=\left(I_{N-1} \otimes A\right) \xi(t)+(\Xi \Sigma \otimes B)\left(I_{N-1} \otimes K\right) \xi(t-\tau(t))$

is asymptotically stable, where $\Xi$ is defined in (13), $\tau(t)=t-t_{k}$, $t_{k} \leq t<t_{k+1}, \forall k \in \mathbb{N}$.

Moreover, the corresponding cost function value (7) is equivalent to:

$J_{e}=\int_{t_{0}}^{\infty}\left\{\xi^{T}(t)\left(\Sigma^{2} \otimes Q\right) \xi(t)\right.$

$$
\left.+\xi^{T}(t-\tau(t))\left(\Sigma^{2} \otimes K^{T} R K\right) \xi(t-\tau(t))\right\} d t,
$$

where the initial state is given as $\xi(t)=$ $\left(Y^{T} \otimes I_{n}\right) x(t), t \in\left[t_{0}-\tau\left(t_{0}\right), t_{0}\right]$.

Proof. Necessity: Let $\xi_{a}(t)=-\left(\frac{\sqrt{N} r^{T} Y}{r^{T} \mathbf{1}_{N}} \otimes I_{n}\right) \xi(t)$, then we have $\left(\frac{r^{T} Y}{\|r\|} \otimes I_{n}\right) \xi(t)+\left(\frac{r^{T} \mathbf{1}_{N}}{\sqrt{N} \cdot\|r\|} \otimes I_{n}\right) \xi_{a}(t) \equiv 0_{n} \quad$ and $\quad\left(\Xi^{-1} \otimes I_{n}\right) \xi(t)=$ $\left(W^{T}\left(I_{N}-\frac{\mathbf{1}_{N} r^{T}}{r^{T} \mathbf{1}_{N}}\right) Y \otimes I_{n}\right) \xi(t)=\left(W^{T} Y \otimes I_{n}\right) \xi(t)+\left(\frac{W^{T} \mathbf{1}_{N}}{\sqrt{N}} \otimes I_{n}\right) \xi_{a}(t)$. By letting $\bar{\xi}(t)=\left[\xi^{T}(t), \xi_{a}^{T}(t)\right]^{T}$, it can be obtained from (14) that
$\left(\Xi^{-1} \otimes I_{n}\right) \dot{\xi}(t)=\left(\Xi^{-1} \otimes A\right) \xi(t)+(\Sigma \otimes B K) \xi(t-\tau(t))$,

and

$$
\begin{aligned}
\left(U V^{T} \otimes I_{n}\right) \dot{\xi}(t)= & \left(U V^{T} \otimes A\right) \bar{\xi}(t) \\
& +\left(U V^{T} V \mathcal{L} V^{T} \otimes B K\right) \bar{\xi}(t-\tau(t)),
\end{aligned}
$$

where $U$ and $V$ are orthogonal matrices defined in (12). Since $\left(U V^{T} \otimes I_{n}\right)$ is invertible, the dynamics of $\bar{\xi}$ can be written as:

$\dot{\xi}(t)=\left(I_{N} \otimes A\right) \xi(t)+\left(V \mathcal{L} V^{T} \otimes B K\right) \xi(t-\tau(t))$.

Since $Y^{T} \mathbf{1}_{N}=\mathbf{0}$, the initial conditions of $\xi(t)$ and $\bar{\xi}(t)$ are $\xi(t)=\left(Y^{T} \otimes I_{n}\right) \delta(t)=\left(Y^{T} \otimes I_{n}\right) x(t), \forall t \in\left[t_{0}-\tau\left(t_{0}\right), t_{0}\right]$ and $\bar{\xi}(t)=$ $\left(V \otimes I_{n}\right) \delta(t), \forall t \in\left[t_{0}-\tau\left(t_{0}\right), t_{0}\right]$, respectively. And by comparing (17) with (10), we conclude that

$\delta(t)=\left(V^{T} \otimes I_{n}\right) \bar{\xi}(t)=\left(V^{T} \otimes I_{n}\right)\left[\begin{array}{c}\xi(t) \\ -\left(\frac{\sqrt{N} r^{T} Y}{r^{T} \mathbf{1}_{N}} \otimes I_{n}\right) \xi(t)\end{array}\right]$.

Since the system (14) is asymptotically stable, i.e., $\lim _{t \rightarrow \infty}\|\xi(t)\|=0$, we get $\lim _{t \rightarrow \infty}\|\delta(t)\|=0$.

Furthermore, let the integrands of (11) and (15) be $f_{1}(t)$ and $f_{2}(t)$, respectively. It is obvious that $f_{1}(t)=f_{2}(t), \forall t \in\left[t_{0}, \infty\right)$, which further implies that $J=\int_{t_{0}}^{\infty} f_{1}(t) d t=\int_{t_{0}}^{\infty} f_{2}(t) d t=J_{e}$. Thus we complete the necessity proof.

Sufficiency: Since we have get that $\delta(t)=\left(V^{T} \otimes I_{n}\right) \bar{\xi}(t)$, where $\bar{\xi}(t)=\left[\xi^{T}(t), \xi_{a}^{T}(t)\right]^{T}$, it follows that $\xi(t)=\left(Y^{T} \otimes I_{n}\right) \delta(t)$. Therefore, $\lim _{t \rightarrow \infty}\|\delta(t)\|=0 \Rightarrow \lim _{t \rightarrow \infty}\|\xi(t)\|=0$, which means that system (14) is asymptotically stable. Moreover, the equivalence between (11) and (15) can be verified by substituting $\bar{\xi}(t)=\left(V \otimes I_{n}\right) \delta(t)$ into (11).

Above all, we complete the proof.

Remark 6. Theorem 1 constructs a novel reduced-order equivalent system to convert the guaranteed cost consensus problem to a guaranteed cost stabilization problem. By dividing the disagreement state-space into two parts, namely, consensus-energy-related subspace and consensus-energy-free subspace, we find the consensus-energy-related state variable $\xi(t)$ and construct the equivalent system. The framework includes the continuous-time and undirected multi-agent systems as special cases, where $\tau(t)=0$ and $\Xi=I$. Note that $J_{e}$ is also in a LQR form, with weighting parameters $\hat{Q}=\Sigma^{2} \otimes Q>0, \hat{R}=\Sigma^{2} \otimes R>0$. Therefore, optimal control results for isolated systems can be directly extended to LTI multi-agent systems by using the reduced-order equivalent system.

With the reduced-order equivalent system, we successfully convert the guaranteed cost consensus problem to the guaranteed cost stabilization problem. By noticing that the equivalent system is modeled by a delay differential equation (DDE) with variable delay, we aim to establish a less conservative stability condition by extending the stability results in [26] to guaranteed cost problems. To this end, LMI technique is used, since the direct Lyapunov functional method for linear DDE always leads to stability conditions in terms of LMIs, and the parametric results can be exploited to optimal protocol design directly.

Theorem 2 (Guaranteed cost consensus condition). Suppose that Assumptions 1 and 2 hold, for given protocol gain $K \in \mathbb{R}^{m \times n}$, guaranteed cost upper bound $\bar{\gamma} \in \mathbb{R}_{+}$, the sampled-data distributed protocol (5) solves the guaranteed cost consensus problem for multiagent system (2) with communication graph $\mathcal{G}$, if there exist $n(N-1) \times n(N-1)$-matrices $\quad P>0, S>0, X, X_{1}, P_{2}, P_{3}, Y_{1}, Y_{2}, Y_{3}$, 
such that LMIs (19)-(21) are feasible:

$\Psi_{1} \triangleq\left[\begin{array}{cc}\Omega_{11} & \Omega_{12} \\ * & \Omega_{22}\end{array}\right]<0, \quad \Psi_{2} \triangleq\left[\begin{array}{cc}\Pi_{11} & \Pi_{12} \\ * & \Pi_{22}\end{array}\right]<0$,

$\Psi_{3} \triangleq\left[\begin{array}{cc}P+h \frac{X+X^{T}}{2} & h X_{1}-h X \\ * & -h X_{1}-h X_{1}^{T}+h \frac{X+X^{T}}{2}\end{array}\right]>0$,

$\Psi_{4} \triangleq \bar{\gamma}-x^{T}\left(t_{0}\right)\left(Y \otimes I_{n}\right) P\left(Y^{T} \otimes I_{n}\right) x\left(t_{0}\right)>0$,

where

$\begin{aligned} \Omega_{11} & =\left[\begin{array}{ccc}\Phi_{11} & \Phi_{12}+h \frac{X+X^{T}}{2} & \Phi_{13} \\ * & \Phi_{22}+h S & \Phi_{23}-h\left(X-X_{1}\right) \\ * & * & \Phi_{33}\end{array}\right], \\ \Pi_{11} & =\left[\begin{array}{cccc}\Phi_{11} & \Phi_{12} & \Phi_{13} & h Y_{1}^{T} \\ * & \Phi_{22} & \Phi_{23} & h Y_{2}^{T} \\ * & * & \Phi_{33} & h Y_{3}^{T} \\ * & * & * & -h S\end{array}\right],\end{aligned}$

$\Omega_{12}=\operatorname{diag}\left\{\Sigma \otimes I_{n}, 0_{n(N-1)}, \Sigma \otimes K^{T}\right\}$,

$\Pi_{12}=\operatorname{diag}\left\{\Sigma \otimes I_{n}, 0_{n(N-1)}, \Sigma \otimes K^{T}, 0_{n(N-1)}\right\}$,

$\Omega_{22}=-\operatorname{diag}\left\{I_{N-1} \otimes Q^{-1}, I_{n(N-1)}, I_{N-1} \otimes R^{-1}\right\}$,

$\Pi_{22}=-\operatorname{diag}\left\{I_{N-1} \otimes Q^{-1}, I_{n(N-1)}, I_{N-1} \otimes R^{-1}, I_{n(N-1)}\right\}$,

$\Phi_{11}=\left(I_{N-1} \otimes A\right)^{T} P_{2}+P_{2}^{T}\left(I_{N-1} \otimes A\right)-Y_{1}-Y_{1}^{T}-\frac{X+X^{T}}{2}$,

$\Phi_{12}=P-P_{2}^{T}+\left(I_{N-1} \otimes A\right)^{T} P_{3}-Y_{2}$,

$\Phi_{13}=Y_{1}^{T}+P_{2}^{T}(\Xi \Sigma \otimes B K)-Y_{3}+X-X_{1}$,

$\Phi_{22}=-P_{3}-P_{3}^{T}, \quad \Phi_{23}=Y_{2}^{T}+P_{3}^{T}(\Xi \Sigma \otimes B K)$,

$\Phi_{33}=Y_{3}+Y_{3}^{T}-\frac{X+X^{T}-2 X_{1}-2 X_{1}^{T}}{2}$.

Proof. Consider the following time-dependent Lyapunov functional for system (14):

$V\left(t, x_{t}, \dot{x}_{t}\right)=\xi^{T}(t) P \xi(t)+V_{s, k}+V_{x, k}, \quad \forall t \in\left[t_{k}, t_{k+1}\right)$,

with $V_{s, k}$ and $V_{x, k}$ defined as:

$V_{s, k}=\left(h_{k}-\tau(t)\right) \int_{t-\tau(t)}^{t} \dot{\xi}^{T}(s) S \dot{\xi}(s) d s$,

$V_{x, k}=\left(h_{k}-\tau(t)\right) \zeta^{T}(t)\left[\begin{array}{cc}\frac{X+X^{T}}{2} & -X+X_{1} \\ * & -X_{1}-X_{1}^{T}+\frac{X+X^{T}}{2}\end{array}\right] \zeta(t)$,

where $t_{k+1}-t_{k}=h_{k} \leq h, \zeta(t)=\operatorname{col}\{\xi(t), \xi(t-\tau(t))\}$. LMI (20) ensures that the positiveness of $V\left(t, x_{t}, \dot{x}_{t}\right)$ holds for $\forall \tau(t) \in[0, h)$, $\forall \zeta(t) \neq \mathbf{0}$. Moreover, since $V_{s, k}$ and $V_{x, k}$ vanish before and after the jump $t=t_{k}, V\left(t, x_{t}, \dot{x}_{t}\right)$ is continuous in time. According to $\dot{\xi}\left(t_{k}\right)=\dot{\xi}(t-\tau(t))=0$ and $\dot{\tau}(t)=1$, we get

$$
\begin{aligned}
\dot{V}_{s, k}= & -\int_{t-\tau(t)}^{t} \dot{\xi}^{T}(s) S \dot{\xi}(s) d s+\left(h_{k}-\tau(t)\right) \dot{\xi}^{T}(t) S \dot{\xi}(t), \\
\dot{V}_{x, k}= & {\left[\begin{array}{c}
2\left(h_{k}-\tau(t)\right) \dot{\xi}(t)-\xi(t) \\
-\xi(t-\tau(t))
\end{array}\right]\left[\begin{array}{cc}
\frac{X+X^{T}}{2} & -X+X_{1} \\
* & -X_{1}-X_{1}^{T}+\frac{X+X^{T}}{2}
\end{array}\right] } \\
& {\left[\begin{array}{c}
\xi(t) \\
\xi(t-\tau(t))
\end{array}\right] . }
\end{aligned}
$$

By using Jensen's inequality $\int_{t-\tau(t)}^{t} \dot{\xi}^{T}(s) S \dot{\xi}(s) d s \geq \tau(t) \nu^{T} S \nu$, $v=\frac{1}{\tau(t)} \int_{t-\tau(t)}^{t} \dot{\xi}(s) d s$, and the following descriptor equation in [30]: $0=2\left[\xi^{T}(t) Y_{1}^{T}+\dot{\xi}^{T}(t) Y_{2}^{T}+\xi^{T}(t-\tau(t)) Y_{3}^{T}\right][-\xi(t)+\xi(t-\tau(t))$

$$
+\tau(t) v]
$$

$0=2\left[\xi^{T}(t) P_{2}^{T}+\dot{\xi}^{T}(t) P_{3}^{T}\right]\left[\left(I_{N-1} \otimes A\right) \xi(t)+(\Xi \Sigma \otimes B K) \xi(t-\tau(t))\right.$

$$
-\dot{\xi}(t)],
$$

it is easy to check that $\frac{d}{d t} V \leq \frac{h-\tau(t)}{h} \cdot \eta_{1}^{T}(t) \Omega_{11} \eta_{1}(t)+\frac{\tau(t)}{h} \cdot \eta_{2}^{T}(t) \Pi_{11} \eta_{2}(t)$, where $\eta_{1}=\operatorname{col}\{\xi(t), \dot{\xi}(t), \xi(t-\tau(t))\}, \eta_{2}=\operatorname{col}\left\{\eta_{1}, v\right\}$.

Since $\Psi_{1}<0$ and $\Psi_{2}<0$, we get $\Omega_{11}-\Omega_{12} \Omega_{22}^{-1} \Omega_{12}^{T}<0$ and $\Pi_{11}-\Pi_{12} \Pi_{22}^{-1} \Pi_{12}^{T}<0$ by Schur complement. Therefore,

$$
\begin{aligned}
& \frac{d}{d t} V+\xi^{T}(t) \hat{Q} \xi(t)+\xi^{T}(t-\tau(t)) \hat{R} \xi(t-\tau(t)) \\
& \leq \frac{h-\tau(t)}{h} \cdot \eta_{1}^{T}(t)\left(\Omega_{11}-\Omega_{12} \Omega_{22}^{-1} \Omega_{12}^{T}\right) \eta_{1}(t) \\
&+\frac{\tau(t)}{h} \cdot \eta_{2}^{T}(t)\left(\Pi_{11}-\Pi_{12} \Pi_{22}^{-1} \Pi_{12}^{T}\right) \eta_{2}(t) \\
&<0, \quad \forall \eta_{2}(t) \neq 0 .
\end{aligned}
$$

From Lyapunov-Krasovskii Theorem, we get $\lim _{t \rightarrow \infty}\|\xi(t)\|=0$, $V(\infty)=0$ and system (14) is asymptotically stable. Thus we conclude from Theorem 1 that the consensus problem is solved. We further integrate both sides of (25) w.r.t. $t$ from $t_{0} \rightarrow \infty$. Since $\tau\left(t_{0}\right)=0$, we then get $V(\infty)-V\left(t_{0}\right) \leq-J_{e}$, which further implies that $\quad J=J_{e} \leq V\left(t_{0}\right) \xi^{T}\left(t_{0}\right) P \xi\left(t_{0}\right)=x^{T}\left(t_{0}\right)\left(Y \otimes I_{n}\right) P\left(Y^{T} \otimes I_{n}\right) x\left(t_{0}\right)$. Therefore, we conclude from $\Psi_{4}>0$ that $J \leq \bar{\gamma}$ by Schur complement, which suffices to complete the proof.

Remark 7. Instead of applying a Lyapunov-Krasovskii analysis to the disagreement dynamics (10) directly, we consider the reducedorder equivalent system (14), so as to abandon redundant variables in the LMI problem without leading to more conservativeness. In the following section, we will show that the equivalent representation is effective in reduced-order guaranteed cost protocol design.

Remark 8. Theorem 2 provides a sufficient condition to verify whether a known protocol gain $K$ solves the guaranteed cost consensus problem. However, the condition is limited in two ways when being exploited to protocol design. First, if $K$ is unknown, LMIs (19) turn to BMIs (bilinear matrix inequalities) due to the existence of the bilinear terms $P_{2}^{T}(\Xi \Sigma \otimes B K)$ and $P_{3}^{T}(\Xi \Sigma \otimes B K)$. Though BMI problems can be solved directly by some modern solvers, LMI-based design scheme seems to be more suitable for large-scale systems due to numerical efficiency. Second, there are too many slack variables in the BMIs, which may lead to high computational complexity.

Remark 9. Note that if regarding $P_{2}$ and $P_{3}$ as tuning parameters, protocol gain $K$ can be computed by solving a resulting LMI. However, it is hard to choose appropriate $P_{2}$ and $P_{3}$.

\section{LMI-based protocol design and optimization}

Since it is hard to synthesize a guaranteed cost consensus 
protocol from Theorem 2, we aim to propose LMI-based protocol design methodologies to parametrize the protocol gain $K$ in this section. Furthermore, some optimization issues are also discussed for completeness.

\subsection{Protocol design for general directed graph case}

We first propose a protocol design methodology for general directed graph case, which is shown in the following theorem.

Theorem 3. Suppose that Assumptions 1 and 2 hold, for given tuning parameters $\epsilon_{1} \in \mathbb{R}_{+}, \beta \in \mathbb{R}$ and guaranteed cost upper bound $\bar{\gamma} \in \mathbb{R}_{+}$, the sampled-data distributed protocol (5) solves the guaranteed cost consensus problem for the multi-agent system (2) with communication graph $\mathcal{G}$, if there exist $n \times n$-matrices $\hat{P}>0, \hat{S}>0, \hat{P}_{2}, \hat{X}, \hat{X}_{1}, \hat{Y}_{1}, \hat{Y}_{2}, \hat{Y}_{3}$ and $\hat{W} \in \mathbb{R}^{m \times n}$, such that the LMIs (26)-(28) are feasible:

$\tilde{\Psi}_{1} \triangleq\left[\begin{array}{cc}\tilde{\Omega}_{11} & \Omega_{12} \\ * & \Omega_{22}\end{array}\right]<0, \quad \tilde{\Psi}_{2} \triangleq\left[\begin{array}{cc}\tilde{\Pi}_{11} & \Pi_{12} \\ * & \Pi_{22}\end{array}\right]<0$,

$\hat{\Psi}_{3} \triangleq\left[\begin{array}{cc}\hat{P}+h \frac{\hat{X}+\hat{X}^{T}}{2} & h \hat{X}_{1}-h \hat{X} \\ * & -h \hat{X}_{1}-h \hat{X}_{1}^{T}+h \frac{\hat{X}+\hat{X}^{T}}{2}\end{array}\right]>0$,

$\hat{\Psi}_{4} \triangleq\left[\begin{array}{cc}\bar{\gamma} & \chi^{T}\left(t_{0}\right)\left(Y \otimes I_{n}\right) \\ * & I_{N-1} \otimes\left(\beta \hat{P}_{2}+\beta \hat{P}_{2}^{T}-\beta^{2} \hat{P}\right)\end{array}\right]>0$,

where

$\tilde{\Omega}_{11}=\left[\begin{array}{ccc}I_{N-1} \otimes \hat{\Phi}_{11} I_{N-1} \otimes\left(\hat{\Phi}_{12}+h \frac{\hat{X}+\hat{X}^{T}}{2}\right) & \tilde{\Phi}_{13} \\ * & I_{N-1} \otimes\left(\hat{\Phi}_{22}+h \hat{S}\right) & \tilde{\Phi}_{23}-I_{N-1} \otimes h\left(\hat{X}-\hat{X}_{1}\right) \\ * & * & I_{N-1} \otimes \hat{\Phi}_{33}\end{array}\right]$,

$\tilde{\Pi}_{11}=\left[\begin{array}{cccc}I_{N-1} \otimes \hat{\Phi}_{11} & I_{N-1} \otimes \hat{\Phi}_{12} & \tilde{\Phi}_{13} & I_{N-1} \otimes h \hat{Y}_{1}^{T} \\ * & I_{N-1} \otimes \hat{\Phi}_{22} & \tilde{\Phi}_{23} & I_{N-1} \otimes h \hat{Y}_{2}^{T} \\ * & * & I_{N-1} \otimes \hat{\Phi}_{33} & I_{N-1} \otimes h \hat{Y}_{3}^{T} \\ * & * & * & -I_{N-1} \otimes h \hat{S}\end{array}\right]$,

$\Omega_{12}=\operatorname{diag}\left\{\Sigma \otimes \hat{P}_{2}^{T}, 0_{n(N-1)}, \Sigma \otimes \hat{W}^{T}\right\}$,

$\Pi_{12}=\operatorname{diag}\left\{\Sigma \otimes \hat{P}_{2}^{T}, 0_{n(N-1)}, \Sigma \otimes \hat{W}^{T}, 0_{n(N-1)}\right\}$,

$\Omega_{22}=-\operatorname{diag}\left\{I_{N-1} \otimes Q^{-1}, I_{n(N-1)}, I_{N-1} \otimes R^{-1}\right\}$,

$\Pi_{22}=-\operatorname{diag}\left\{I_{N-1} \otimes Q^{-1}, I_{n(N-1)}, I_{N-1} \otimes R^{-1}, I_{n(N-1)}\right\}$,

$\hat{\Phi}_{11}=\hat{P}_{2}^{T} A^{T}+A \hat{P}_{2}-\hat{Y}_{1}-\hat{Y}_{1}^{T}-\frac{\hat{X}+\hat{X}^{T}}{2}$,

$\hat{\Phi}_{12}=\hat{P}-\hat{P}_{2}+\epsilon_{1} \hat{P}_{2}^{T} A^{T}-\hat{Y}_{2}$,

$\tilde{\Phi}_{13}=I_{N-1} \otimes\left(\hat{Y}_{1}^{T}-\hat{Y}_{3}+\hat{X}-\hat{X}_{1}\right)+(\Xi \Sigma) \otimes B \hat{W}$,

$\hat{\Phi}_{22}=-\epsilon_{1} \hat{P}_{2}-\epsilon_{1} \hat{P}_{2}^{T}, \quad \tilde{\Phi}_{23}=I_{N-1} \otimes \hat{Y}_{2}^{T}+\epsilon_{1}(\Xi \Sigma) \otimes B \hat{W}$,

$\hat{\Phi}_{33}=\hat{Y}_{3}+\hat{Y}_{3}^{T}-\frac{\hat{X}+\hat{X}^{T}-2 \hat{X}_{1}-2 \hat{X}_{1}^{T}}{2}$.
Moreover, the protocol gain is $K=\hat{W} \hat{P}_{2}^{-1}$.

Proof. Before we prove the guaranteed cost stability for the whole equivalent system, we should notice that $\hat{P}_{2}$ is nonsingular. If $\hat{P}_{2}$ is singular, then there exists $z \neq \mathbf{0}$ such that $z^{T}\left(I_{N-1} \otimes \hat{\Phi}_{22}\right) z=0$, which leads to a contradiction.

Then construct a refined time-dependent Lyapunov functional candidate $V\left(t, x_{t}, \dot{x}_{t}\right)$ for the equivalent system as (24), with its parameters chosen as,

$P=I_{N-1} \otimes \bar{P}, \quad P_{2}=I_{N-1} \otimes \bar{P}_{2}, \quad P_{3}=I_{N-1} \otimes \bar{P}_{3}$,

$S=I_{N-1} \otimes \bar{S}, \quad X=I_{N-1} \otimes \bar{X}, \quad X_{1}=I_{N-1} \otimes \bar{X}_{1}$,

$Y_{1}=I_{N-1} \otimes \bar{Y}_{1}, \quad Y_{2}=I_{N-1} \otimes \bar{Y}_{2}, \quad Y_{3}=I_{N-1} \otimes \bar{Y}_{3}$,

$\left\{\bar{P}, \bar{P}_{3}, \bar{S}, \bar{X}, \bar{X}_{1}, \bar{Y}_{1}, \bar{Y}_{2}, \bar{Y}_{3}\right\}=\bar{P}_{2}^{T}\left\{\hat{P}, \hat{P}_{3}, \hat{S}, \hat{X}, \hat{X}_{1}, \hat{Y}_{1}, \hat{Y}_{2}, \hat{Y}_{3}\right\} \bar{P}_{2}$

$\bar{P}_{2}=\hat{P}_{2}^{-1}, \quad \hat{P}_{3}=\epsilon_{1} \hat{P}_{2}$,

where $\hat{P}, \hat{S}, \hat{P}_{2}, \hat{X}, \hat{X}_{1}, \hat{Y}_{1}, \hat{Y}_{2}, \hat{Y}_{3}$ are a set of feasible solution to the LMIs (26)-(28).

By Theorem 2, the guaranteed cost consensus problem is solved if $V\left(t, x_{t}, \dot{x}_{t}\right)$ is a Lyapunov functional for the equivalent system and satisfies (25). Since the condition has been formulated in terms of LMIs (19) and (20), we will turn to prove that $\Psi_{1}<0, \Psi_{2}<0, \Psi_{3}>0$ hold with parameters (31) to avoid repetition.

To this end, we first deduce from (26) that $\Psi_{1}<0, \Psi_{2}<0$ since the congruence relations $\Psi_{1}=T_{1}^{T} \tilde{\Psi}_{1} T_{1}, \Psi_{2}=T_{2}^{T} \tilde{\Psi}_{1} T_{2}$ hold for $T_{1}=\operatorname{diag}\left\{P_{2}, P_{2}, P_{2}, I_{2 n(N-1)}, I_{m(N-1)}\right\}$, $T_{2}=\operatorname{diag}\left\{P_{2}, P_{2}, P_{2}, P_{2}, I_{2 n(N-1)}, I_{m(N-1)}, I_{n(N-1)}\right\}$.

On the other hand, $\hat{\Psi}_{3}>0$ implies that $\varsigma_{i}^{T} \hat{\Psi}_{3}^{(i)} \varsigma_{i}>0$ for $\forall \varsigma_{i} \in \mathbb{R}^{2 n}, \varsigma_{i} \neq \mathbf{0}, i=2, \ldots, N$, where $\hat{\Psi}_{3}^{(i)}=\hat{\Psi}_{3}, \varsigma_{i}=\operatorname{col}\left\{\varsigma_{i 1}, \varsigma_{i 2}\right\}$ with $\varsigma_{i 1}, \varsigma_{i 2} \in \mathbb{R}^{n}$. Then let $\varsigma=\operatorname{col}\left\{\varsigma^{(1)}, \varsigma^{(2)}\right\}$ with $\varsigma^{(i)}=\operatorname{col}\left\{\varsigma_{2 i}, \ldots, \varsigma_{(N-1) i}\right\}$, it is easy to check that

$\varsigma^{T}\left(T_{3}^{T} \Psi_{3} T_{3}\right) \varsigma=\sum_{i=2}^{N-1}\left\{\varsigma_{i}^{T} \stackrel{\leftrightarrow}{\Psi}_{1}^{(i)} \varsigma_{i}\right\}>0, \quad$ for $\forall \varsigma \neq \mathbf{0}$,

where $T_{3}=\operatorname{diag}\left\{P_{2}^{-1}, P_{2}^{-1}\right\}$. Therefore, $T_{3}^{T} \Psi_{3} T_{3}>0$ and $\Psi_{3}>0$.

Finally, we show that the cost is upper bounded by $\bar{\gamma}$. Since $\left(\hat{P}_{2}-\beta \hat{P}\right) \hat{P}^{-1}\left(\hat{P}_{2}-\beta \hat{P}\right)^{T} \geq 0, \quad \hat{P}>0$ implies that $\hat{P}_{2} \hat{P}^{-1} \hat{P}_{2}^{T} \geq \beta \hat{P}_{2}+$ $\beta \hat{P}_{2}^{T}-\beta^{2} \hat{P}$, we conclude that

$$
\begin{gathered}
{\left[\begin{array}{cc}
\bar{\gamma} & x^{T}\left(t_{0}\right)\left(Y \otimes I_{n}\right) \\
* & I_{N-1} \otimes \hat{P}_{2} \hat{P}^{-1} \hat{P}_{2}^{T}
\end{array}\right] \geq \hat{\Psi}_{4}>0 \text { and }} \\
\quad J=x\left(t_{0}\right)\left(Y Y^{T} \otimes \hat{P}_{2}^{-T} \hat{P} \hat{P}_{2}^{-1}\right) x\left(t_{0}\right) \leq \bar{\gamma},
\end{gathered}
$$

which suffices to complete the proof.

Remark 10. In fact, $Y$ is not required to establish LMI (28). By noting that $Y Y^{T}=I_{N}-\frac{\mathbf{1}_{N} \mathbf{1}_{N}^{T}}{N}=Z Z^{T}$ holds for $\forall Z \in \mathbb{R}^{N \times(N-1)}$ satisfying $Z^{\mathrm{T}} \mathbf{1}_{N}=\mathbf{0}$ and $Z^{\mathrm{T}} Z=I_{N-1}$, LMI (28) is equivalent to:

$\hat{\Psi}_{4} \triangleq\left[\begin{array}{cc}\bar{\gamma} & x^{T}\left(t_{0}\right)\left(Z \otimes I_{n}\right) \\ * & I_{N-1} \otimes\left(\beta \hat{P}_{2}+\beta \hat{P}_{2}^{T}-\beta^{2} \hat{P}\right)\end{array}\right]>0$

for $Z^{\mathrm{T}} \mathbf{1}_{N}=\mathbf{0}$ and $Z^{\mathrm{T}} Z=I_{N-1}$. Furthermore, we can use GramSchmidt process to construct a set of orthonormal basis $\frac{\mathbf{1}_{N}}{\sqrt{N}}, e_{1}, \ldots, e_{N-1}$ for the space $\mathbb{R}^{N}$, then an optional $Z$ can be chosen 
as $Z=\left[e_{1}, \ldots, e_{N-1}\right]$.

Remark 11. To determine the guaranteed cost consensus protocol for a directed multi-agent system using Theorem 3, we need to know its graph information $\Xi, \Sigma$ and the initial states of all agents $x_{i}\left(t_{0}\right)$. However, it is not limited, since once the protocol is assigned to each agent, the multi-agent system can achieve prescribed robust performance by using relative information only.

\subsection{Reduced-order protocol design for undirected graph case via decomposition}

Undirected graphs can be regarded as a special kind of directed graphs such that all the edges are bidirectional. They are important in guaranteed cost consensus problems, since they have the nice property that $\Xi=I$. We show that, for undirected graph case, the equivalent system can be further decomposed into $N-1$ decoupled subsystems, whose guaranteed cost stabilities lead to guaranteed cost consensus of the multi-agent system. To further reduce the computational complexity and the global information required, we propose a reduced-order protocol design method for undirected graph case, where only the largest and smallest singular values of graph Laplacian are required.

Since $\mathcal{G}$ is undirected, we deduce from Lemma 2 that $\Xi=I_{N-1}$, and the equivalent system is

$\dot{\xi}(t)=\left(I_{N-1} \otimes A\right) \xi(t)+(\Sigma \otimes B K) \xi(t-\tau(t))$,

$J_{e}=\int_{t_{0}}^{\infty}\left\{\xi^{T}(t)\left(\Sigma^{2} \otimes Q\right) \xi(t)+\xi^{T}(t-\tau(t))\left(\Sigma^{2} \otimes K^{T} R K\right) \xi(t-\tau(t))\right\} d t$,

which can be further decomposed into $N-1$ decoupled subsystems:

$\dot{\xi}_{i}(t)=A \xi_{i}(t)+\sigma_{i} B K \xi_{i}(t-\tau(t))$,

$J_{i}=\sigma_{i}^{2} \int_{t_{0}}^{\infty}\left\{\xi_{i}^{T}(t) Q \xi_{i}(t)+\xi_{i}^{T}(t-\tau(t)) K^{T} R K \xi_{i}(t-\tau(t))\right\} d t$,

where $\quad i=2, \ldots, N, \quad \xi_{i} \in \mathbb{R}^{n}, \quad \xi=\operatorname{col}\left\{\xi_{1}, \ldots, \xi_{N}\right\}, \quad \xi(t)=$ $\left(Y^{T} \otimes I_{n}\right) x(t), t \in\left[t_{0}-\tau\left(t_{0}\right), t_{0}\right]$.

The connection between guaranteed cost consensus and guaranteed cost stabilization of these subsystems is indicated in the following lemma.

Lemma 3. Suppose that Assumptions 1 and 2 hold, the distributed protocol (5) solves the guaranteed cost consensus problem for the multi-agent system (2) with undirected communication graph $\mathcal{G}$, if for $i=2, \ldots, N$, all the subsystems (34) are asymptotically stable, and the corresponding cost function values satisfy $J_{i} \leq J_{i}^{*}$. Moreover, the cost function (7) satisfies $J \leq \sum_{2}^{N} J_{i}^{*}$.

Proof. From the asymptotic stability of the subsystems, we get that $\lim _{t \rightarrow \infty}\left\|\xi_{i}(t)\right\|=0, i=2, \ldots, N$ and $\lim _{t \rightarrow \infty}\|\xi(t)\|=0$. Furthermore, $J_{e}=\sum_{2}^{N} J_{i} \leq \Sigma_{2}^{N} J_{i}^{*}$. Then we conclude from Theorem 1 and Definition 3 that guaranteed cost consensus is achieved, which suffices to complete the proof.

With the idea of decomposition, the protocol design problem can be solved by the following theorem, where only the largest and smallest singulars of $\mathcal{L}$ are required.

Theorem 4. Suppose that Assumptions 1 and 2 hold, for given tuning parameter $\epsilon_{1} \in \mathbb{R}_{+}, \beta \in \mathbb{R}$ and guaranteed cost upper bound $\bar{\gamma} \in \mathbb{R}_{+}$, the sampled-data distributed protocol (5) solves the guaranteed cost consensus problem for multi-agent system (2) with undirected communication graph $\mathcal{G}$, if there exist $n \times n$-matrices $\hat{P}>0, \hat{S}>0, \hat{P}_{2}, \hat{X}, \hat{X}_{1}, \hat{Y}_{1}, \hat{Y}_{2}, \hat{Y}_{3}$ and $\hat{W} \in \mathbb{R}^{m \times n}$ satisfying (27), (32), and (35):
$\hat{\Psi}_{1}^{(i)} \triangleq\left[\begin{array}{cc}\hat{\Omega}_{11}^{(i)} & \hat{\Omega}_{12}^{(i)} \\ * & \hat{\Omega}_{22}\end{array}\right]<0, \quad \hat{\Psi}_{2}^{(i)} \triangleq\left[\begin{array}{cc}\hat{\Pi}_{11}^{(i)} & \hat{\Pi}_{12}^{(i)} \\ * & \hat{\Pi}_{22}\end{array}\right]<0, \quad i=2, N$,

where

$\hat{\Omega}_{11}^{(i)}=\left[\begin{array}{ccc}\hat{\Phi}_{11} & \hat{\Phi}_{12}+h \frac{\hat{X}+\hat{X}^{T}}{2} & \hat{\Phi}_{13}^{(i)} \\ * & \hat{\Phi}_{22}+h \hat{S} & \hat{\Phi}_{23}^{(i)}-h\left(\hat{X}-\hat{X}_{1}\right) \\ * & * & \hat{\Phi}_{33}\end{array}\right]$,

$\hat{\Pi}_{11}^{(i)}=\left[\begin{array}{cccc}\hat{\Phi}_{11} & \hat{\Phi}_{12} & \hat{\Phi}_{13}^{(i)} & h \hat{Y}_{1}^{T} \\ * & \hat{\Phi}_{22} & \hat{\Phi}_{23}^{(i)} & h \hat{Y}_{2}^{T} \\ * & * & \hat{\Phi}_{33} & h \hat{Y}_{3}^{T} \\ * & * & * & -h \hat{S}\end{array}\right]$,

$\hat{\Omega}_{12}^{(i)}=\operatorname{diag}\left\{\sigma_{i} \hat{P}_{2}^{T}, 0_{n}, \sigma_{i} \hat{W}^{T}\right\}, \quad \hat{\Pi}_{12}^{(i)}=\operatorname{diag}\left\{\sigma_{i} \hat{P}_{2}^{T}, 0_{n}, \sigma_{i} \hat{W}^{T}, 0_{n}\right\}$,

$\hat{\Omega}_{22}=-\operatorname{diag}\left\{Q^{-1}, I_{n}, R^{-1}\right\}, \quad \hat{\Pi}_{22}=-\operatorname{diag}\left\{Q^{-1}, I_{n}, R^{-1}, I_{n}\right\}$,

$\hat{\Phi}_{13}^{(i)}=\hat{Y}_{1}^{T}+\sigma_{i} B \hat{W}-\hat{Y}_{3}+\hat{X}-\hat{X}_{1}, \quad \hat{\Phi}_{23}^{(i)}=\hat{Y}_{2}^{T}+\epsilon_{1} \sigma_{i} B \hat{W}$,

and $\hat{\Phi}_{11}, \hat{\Phi}_{12}, \hat{\Phi}_{22}, \hat{\Phi}_{33}$ are defined in (30). Moreover, the protocol gain is $K=\hat{W} \hat{P}_{2}^{-1}$.

Proof. Since $\bar{P}_{2}$ is nonsingular, which can be easily proved by contradiction, we let $\bar{P}_{2}=\hat{P}_{2}^{-1}, K=\hat{W} \bar{P}_{2}$,

$\left\{\bar{P}, \bar{S}, \bar{X}, \bar{X}_{1}, \bar{Y}_{1}, \bar{Y}_{2}, \bar{Y}_{3}\right\}=\bar{P}_{2}^{T}\left\{\hat{P}, \hat{S}, \hat{X}, \hat{X}_{1}, \hat{Y}_{1}, \hat{Y}_{2}, \hat{Y}_{3}\right\} \bar{P}_{2}$,

$D_{1}=\operatorname{diag}\left\{\bar{P}_{2}, \bar{P}_{2}, \bar{P}_{2}\right\}, \quad D_{2}=\operatorname{diag}\left\{\bar{P}_{2}, \bar{P}_{2}, \bar{P}_{2}, \bar{P}_{2}\right\}$.

Next, we will prove that the $i$-th subsystem (34) achieves guaranteed cost stabilization. To this end, we take the following Lyapunov functional candidate for $i=2, \ldots, N$ :

$V^{(i)}=\xi_{i}^{T}(t) \bar{P} \xi_{i}(t)+\left(h_{k}-\tau(t)\right) V_{s x}^{(i)}, \forall t \in\left[t_{k}, t_{k+1}\right)$,

with the term $V_{s x}^{(i)}$ defined as follows:

$V_{s x}^{(i)}=\int_{t-\tau(t)}^{t} \dot{\xi}_{i}^{T}(s) \bar{S} \dot{\xi}_{i}(s) d s$

$+\zeta_{i}^{T}(t)\left[\begin{array}{cc}\frac{\bar{X}+\bar{X}^{T}}{2} & -\bar{X}+\bar{X}_{1} \\ * & -\bar{X}_{1}-\bar{X}_{1}^{T}+\frac{\bar{X}+\bar{X}^{T}}{2}\end{array}\right] \zeta_{i}(t)$,

where $\zeta_{i}(t)=\operatorname{col}\left\{\xi_{i}(t), \xi_{i}(t-\tau(t))\right\}$. The positiveness of $V^{(i)}$ for $\forall \tau(t) \in[0, h)$ can be checked by analyzing $\hat{\Psi}_{3}(27)$. Then we take the same procedure in Theorem 2 to get $\dot{V}^{(i)}$. It is clear that $V^{(i)}$ is continuous in time, and we can obtain from Jensen's inequality (1) and descriptor equations:

$$
\begin{aligned}
0= & 2\left[\xi_{i}^{T}(t) \hat{Y}_{1}^{T}+\dot{\xi}_{i}^{T}(t) \hat{Y}_{2}^{T}+\xi_{i}^{T}(t-\tau(t)) \hat{Y}_{3}^{T}\right]\left[-\xi_{i}(t)+\xi_{i}(t-\tau(t))\right. \\
& \left.+\tau(t) v_{i}\right], \\
0= & 2\left[\xi_{i}^{T}(t) \hat{P}_{2}^{T}+\dot{\xi}_{i}^{T}(t) \hat{P}_{3}^{T}\right]\left[\left(I_{N-1} \otimes A\right) \xi_{i}(t)+(\Xi \Sigma \otimes B K) \xi_{i}(t-\tau(t))\right. \\
& \left.-\dot{\xi}_{i}(t)\right],
\end{aligned}
$$

that 


$$
\begin{aligned}
\frac{d}{d t} V^{(i)}+\sigma_{i}^{2} \cdot\left\{\xi_{i}^{T}(t) Q \xi_{i}(t)+\xi_{i}^{T}(t-\tau(t)) R \xi_{i}(t-\tau(t))\right\} \\
\leq \frac{h-\tau(t)}{h} \cdot y_{i}^{T}(t) \hat{\Psi}_{1}^{(i)} y_{i}(t)+\frac{\tau(t)}{h} \cdot z_{i}^{T}(t) \hat{\Psi}_{2}^{(i)} z_{i}(t) \\
=\frac{h-\tau(t)}{h} \cdot y_{i}^{T}(t)\left[\alpha_{i} \cdot \hat{\Psi}_{1}^{(N)}+\left(1-\alpha_{i}\right) \cdot \hat{\Psi}_{1}^{(2)}\right] y_{i}(t) \\
\quad+\frac{\tau(t)}{h} \cdot z_{i}^{T}(t)\left[\alpha_{i} \cdot \hat{\Psi}_{2}^{(N)}+\left(1-\alpha_{i}\right) \cdot \hat{\Psi}_{2}^{(2)}\right] z_{i}(t) \\
<0, \quad \forall z_{i} \neq 0,
\end{aligned}
$$

where $\quad \alpha_{i}=\frac{\sigma_{i}-\sigma_{2}}{\sigma_{N}-\sigma_{2}} \in[0,1], \quad y_{i}=T_{1}^{(i)} \cdot \operatorname{col}\left\{\xi_{i}(t), \dot{\xi}_{i}(t), \xi_{i}(t-\tau(t)), \mathbf{0}_{3 n}\right\}$, $z_{i}=T_{2}^{(i)} \cdot \operatorname{col}\left\{\xi_{i}(t), \dot{\xi}_{i}(t), \xi_{i}(t-\tau(t)), v_{i}, \mathbf{0}_{4 n}\right\}, \quad v_{i}=\frac{1}{\tau(t)} \int_{t-\tau(t)}^{t} \dot{\xi}_{i}(s) d s, \quad \hat{P}_{3}=\epsilon_{1} \hat{P}_{2}$, $T_{1}^{(i)}$ and $T_{2}^{(i)}$ are full-rank linear transformation matrices for the $i$-th subsystem such that

$T_{1}^{(i)}=\left[\begin{array}{cc}D_{1}^{T} & -D_{1}^{T} \hat{\Omega}_{12}^{(i)} \hat{\Omega}_{22}^{-1} \\ 0 & I_{3 n}\end{array}\right]^{T}$,

$T_{2}^{(i)}=\left[\begin{array}{cc}D_{2}^{T} & -D_{2}^{T} \hat{\Pi}_{12}^{(i)} \hat{\Pi}_{22}^{-1} \\ 0 & I_{4 n}\end{array}\right]^{T}$.

By noting that $\frac{d}{d t} V^{(i)}<0, \forall z_{i}(t) \neq 0$ and integrating both sides of (38) w.r.t. $t$ from $t_{0} \rightarrow \infty$, we get $\lim _{t \rightarrow \infty}\left\|\xi_{i}(t)\right\|=0$ and $J_{i} \leq V^{(i)}\left(t_{0}\right)=\xi_{i}^{T}\left(t_{0}\right) \bar{P} \xi_{i}\left(t_{0}\right)=J_{i}^{*}$, where $\operatorname{col}\left\{\xi_{2}\left(t_{0}\right), \ldots, \xi_{N}\left(t_{0}\right)\right\}=$ $\left(Y^{T} \otimes I_{n}\right) \delta\left(t_{0}\right)=\left(Y^{T} \otimes I_{n}\right) x\left(t_{0}\right)$. Then we conclude from Lemma 3 and Remark 10 that guaranteed cost consensus is achieved with $K=\hat{W} \hat{P}_{2}^{-1}$ and

$J \leq \sum_{2}^{N} J_{i}^{*}=x^{T}\left(t_{0}\right)\left(Y Y^{T} \otimes \hat{P}_{2}^{-T} \hat{P} \hat{P}_{2}^{-1}\right) x\left(t_{0}\right)$

$\leq x^{T}\left(t_{0}\right)\left(Z Z^{T} \otimes\left(\beta \hat{P}_{2}+\beta \hat{P}_{2}^{T}-\beta^{2} \hat{P}\right)^{-1}\right) x\left(t_{0}\right)<\bar{\gamma}$,

which suffices to complete the proof.

Remark 12. Compared to Theorems 2 and 3, Theorem 4 only requires $\sigma_{2}, \sigma_{N}$ and $x_{i}\left(t_{0}\right), i=1, \ldots, N$, to determine the guaranteed cost consensus protocol. Therefore, the global information required in design is reduced.

Remark 13. In Theorems 3 and 4 , we let $\hat{P}_{3}=\epsilon_{1} \hat{P}_{2}$ and use (32) to bound the cost, though lead to some conservativeness, they reduce the computation burden and simplify the parameter selection procedure. Note that, compared to BMIs (19), LMIs (35) are lower order LMIs with fewer decision variables. See Table 1 for numerical complexity and some other properties of the above methods. In Table 1 , it is obvious that the protocol design methods based on Theorems 3 and 4 become more and more efficient with the increasing of the agent number $N$.

\subsection{Protocol optimization issues}

Based on the parametric results proposed in Theorems 2-4, we

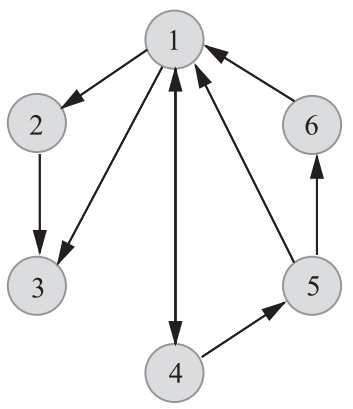

Fig. 1. Directed communication topology of Example 1.

further discuss some optimization issues on protocol design.

\subsubsection{Sub-optimal protocol design}

To readily find the sub-optimal guaranteed cost consensus protocol $K^{*}$ for (2) with sub-minimum cost bound $\bar{\gamma}^{*}$, the following SDP problems are established for directed and undirected graph cases, respectively:

SDP-1: ( $G$ is directed) Minimize $\bar{\gamma}$, subject to (26), (27), and (28) for given $\epsilon_{1}, \beta$.

SDP-2: ( $\mathcal{G}$ is undirected) Minimize $\bar{\gamma}$, subject to (27), (32), and (35) for given $\epsilon_{1}, \beta$.

Remark 14. By solving SDP-1 or SDP-2, we can get the optimal cost bound $\bar{\gamma}^{*}$, the optimized decision variables $\hat{W}^{*}$ and $\hat{P}_{2}^{*}$, and the sub-optimal protocol gain $K^{*}=\hat{W}^{*}\left(\hat{P}_{2}^{*}\right)^{-1}$. Since Theorems 3 and 4 only provide sufficient conditions for guaranteed cost consensus, the resulting protocol gain $K^{*}$ is only sub-optimal.

\subsubsection{Enlarging allowable sampling interval bound}

With the increasing of the sampling interval, the sampling times during a fixed time interval may decrease, which means that less remote sensing and interaction reliability are required to perform sampled-data guaranteed cost consensus. Therefore, we are interested in enlarging sampling interval bound $h$ that preserves the guaranteed cost consensus property for the sampleddata multi-agent system.

For the given guaranteed cost consensus protocol gain $K$ and guaranteed cost bound $\bar{\gamma}$, according to Theorem 2, the sampling interval bound $h$ can be enlarged by solving the following bilinear SDP problem (or BMI problem),

BMI-SDP: Maximize $h$, subject to (19)-(21) for given $K, \bar{\gamma}$.

Remark 15. Due to the cross terms $h \hat{X}, h \hat{X}_{1}, h \hat{Y}_{1}, h \hat{Y}_{2}, h \hat{Y}_{3}, h S$, the constraints of the BMI-SDP are in bilinear matrix forms. However, by noting that $h$ is a scalar variable, and (19)-(21) turn to LMIs for a fixed $h$, we propose an iterative LMI algorithm to solve the BMI-

\begin{tabular}{|c|c|c|c|c|c|c|}
\hline Method & Graph information required & Tuning parameters & Number of decision variables & Number of LMIs & The maximum order of LMIs & Graph type \\
\hline Theorem 2 & $\Xi, \Sigma$ & $P_{2}, P_{3}$ & $6 n^{2}(N-1)^{2}+n(N-1)+m n$ & 4 & $(7 n+m)(N-1)$ & Directed \\
\hline Theorem 3 & $\Xi, \Sigma$ & $\epsilon_{1}, \beta$ & $7 n^{2}+n+m n$ & 4 & $(7 n+m)(N-1)$ & Directed \\
\hline Theorem 4 & $\sigma_{2}, \sigma_{N}$ & $\epsilon_{1}, \beta$ & $7 n^{2}+n+m n$ & 6 & $7 n+m$ & Undirected \\
\hline
\end{tabular}
SDP, based on the well-known binary search algorithm.

\section{Algorithm 1.}

Table 1

Numerical complexity of different protocol design methods. 

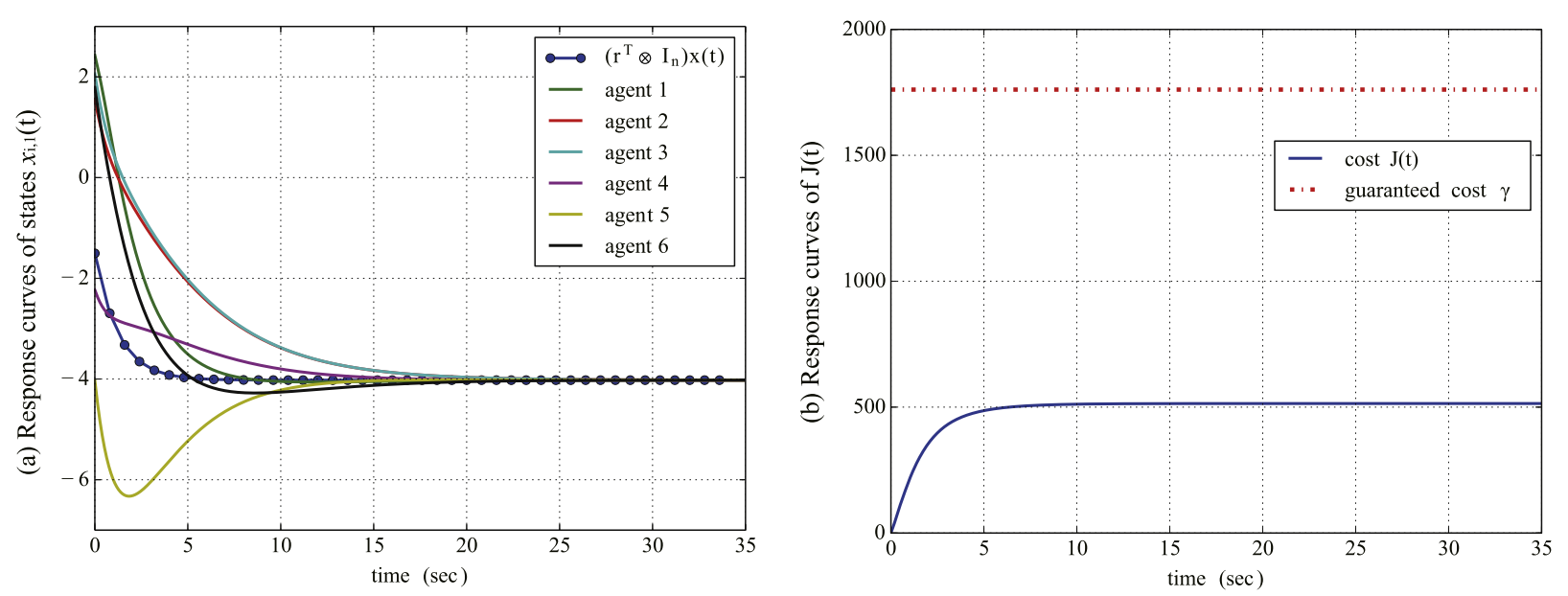

Fig. 2. Consensus process of Example 1 with $h=0.5$. (a) State trajectories and (b) energy consumption.
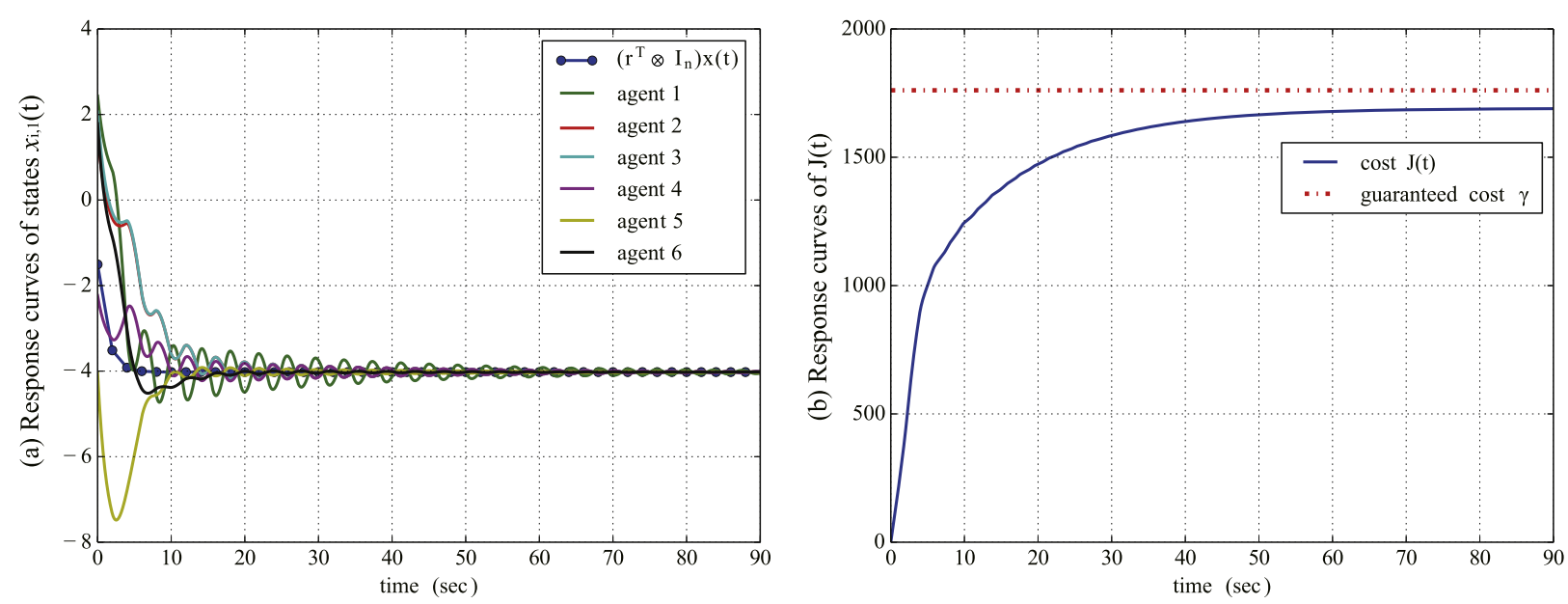

Fig. 3. Guaranteed cost consensus is achieved for Example 1 with $h=1.95$. (a) State trajectories and (b) energy consumption.
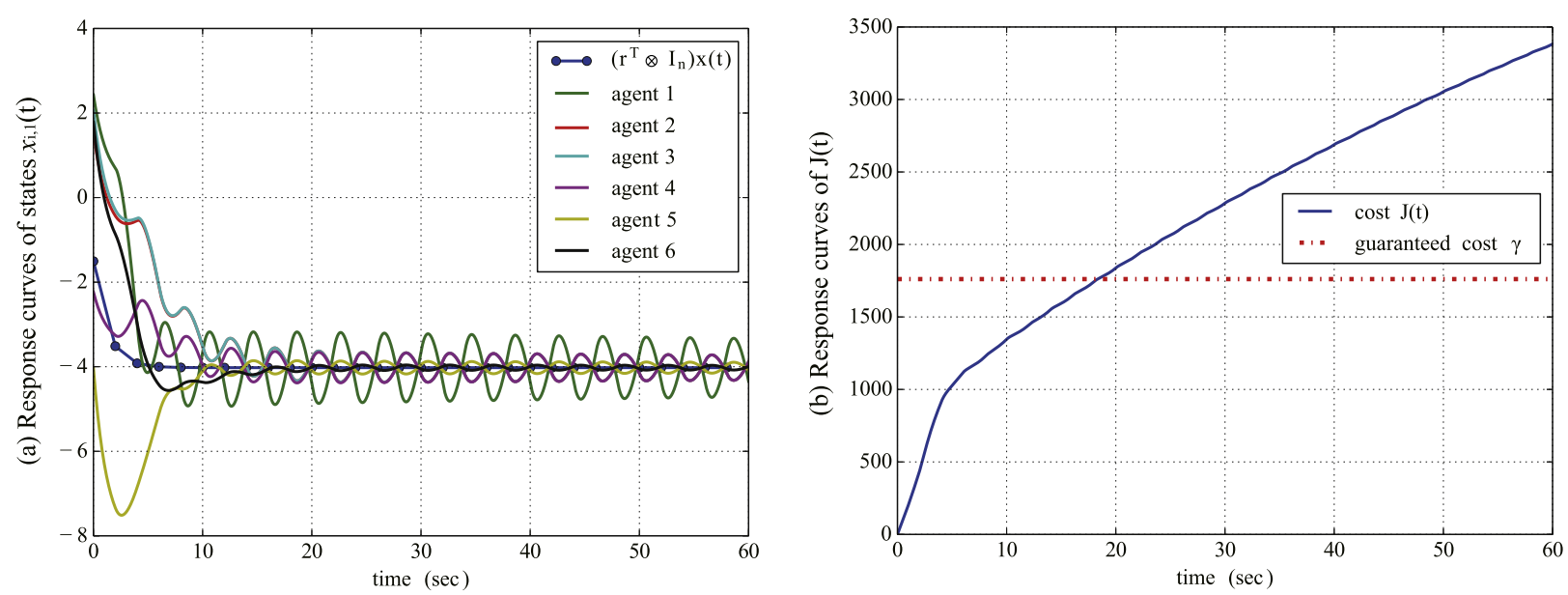

Fig. 4. Guaranteed cost consensus cannot be achieved for Example 1 with $h=2.00$. (a) State trajectories and (b) energy consumption.

Step 1: Set the initial state $x_{i}(0), i \in \mathcal{I}$, model parameters $A, B, Q, R, \Xi, \Sigma$, protocol gain $K$, guaranteed cost bound $\bar{\gamma}$ and the computational accuracy $\epsilon$.

Step 2: Find a feasible solution $h_{L}>0$, such that LMIs (19)-(21) are feasible for $h=h_{L}$. Set $h_{H}=h_{L}$.

Step 3: Set $h_{H}=2 h_{H}$. If LMIs (19)-(21) are feasible for $h=h_{H}$, go to Step 3. Otherwise, go to Step 4 .

Step 4: Set $h_{M}=\left(h_{L}+h_{H}\right) / 2$. If LMIs (19)-(21) are feasible for
$h=h_{M}$, set $h_{L}=h_{M}$. Otherwise, set $h_{H}=h_{M}$.

Step 5: If $\left|h_{H}-h_{L}\right|<\epsilon$, output $\bar{h}=h_{L}$. Otherwise, go to Step 4.

Remark 16. In Step 3, we aim to get an $h_{H}$ such that LMIs (19)-(21) are infeasible for given $K$ and $\bar{\gamma}$, so that the upper bound of allowable sampling interval $\bar{h}$ is contained in $\left[h_{L}, h_{H}\right)$. In Step 4 , binary search algorithm is used to estimate $\bar{h}$, whose correctness can be verified by induction. To be more specific, let $l^{(i)}=h_{H}-h_{L}$, 


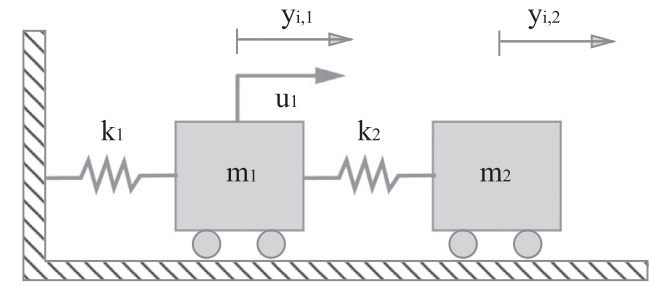

Fig. 5. Two-mass-spring system as an agent in Example 2.

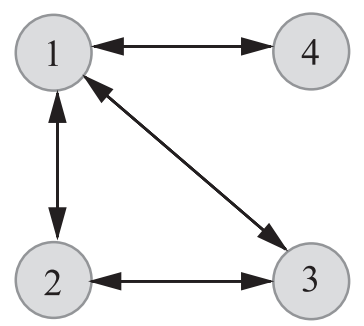

Fig. 6. Undirected communication topology of Example 2.

$\bar{h}^{(i)}=h_{L}$ in the $\{i\}$-th iteration of Step 4 . Then in the $\{i+1\}$-th iteration of Step 4 , we get $l^{(i+1)}=0.5 \times l^{(i)}, \bar{h}^{(i+1)} \geq \bar{h}^{(i)}$. Since LMIs (19)-(21) are feasible with $h=\bar{h}^{(i)}, \bar{h}^{(i+1)} \geq \bar{h}^{(i)}$ and $\left|\bar{h}^{(i)}-\bar{h}\right| \leq l^{(i)}$ hold in each iteration of Step 4, Step 5 then renders that the iterative process of Step 4 stops when the estimation error is less than the accuracy level $\epsilon$.

\section{Simulations}

In this section, two numerical examples are provided to validate the effectiveness of our theoretical results.

Example 1 (Protocol design for directed graph case using Theorem $3)$. Consider a sampled-data multi-agent system consisting of $N=6$ agents, the agent dynamics of which can be described by (2) with $A, B, Q, R$ given as

$A=\left[\begin{array}{cc}0 & 1 \\ 0 & -0.8\end{array}\right], \quad B=\left[\begin{array}{c}0 \\ 0.5\end{array}\right], \quad Q=\left[\begin{array}{cc}0.9 & 0 \\ 0 & 0.9\end{array}\right], \quad R=1.2$.

The sampling instants satisfy $t_{k+1}-t_{k} \leq h=0.5 \mathrm{~s}, \forall k \in \mathbb{N}$.

In the simulation, the initial state of each agent is generated randomly in $[-5,5]$, and $\mathcal{G}$ is described in Fig. 1 . Next, we aim to design sub-optimal guaranteed cost consensus protocol (5) for the multi-agent system. By solving SDP- 1 for given $\epsilon_{1}=1, \beta=1.0$ via

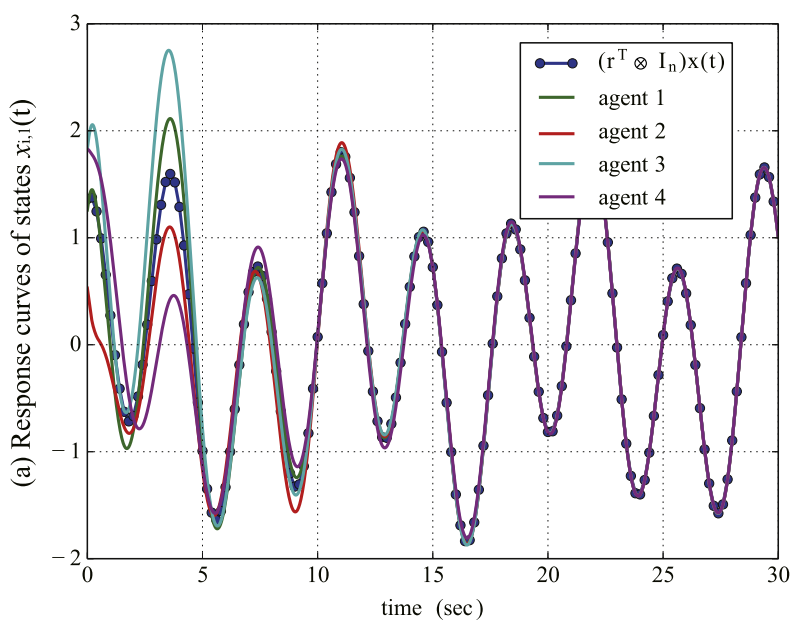

YALMIP [31] and Sedumi solver, we obtain

$K^{*}=\left[\begin{array}{ll}-0.3787 & -0.6237\end{array}\right], \quad \bar{\gamma}^{*}=1761.0$.

The response curves of states and cost are shown in Fig. 2, from which we conclude that guaranteed cost consensus is achieved. Furthermore, by letting computational accuracy $\epsilon=0.01$, the allowable sampling interval bound is enlarged to $\bar{h}=1.95$ by Algorithm 1.

By applying standard techniques from digital control in simulations, the closed-loop system remains consensus for all samplings satisfying $h \leq 2.00$ and achieves guaranteed cost consensus for $h \leq 1.95$. We then let the sampling intervals be fixed at $h_{k}=1.95$ and $h_{k}=2.00$ in simulations, with system response curves shown in Figs. 3 and 4, respectively. We find that guaranteed cost consensus is achieved with $h_{k}=1.95$, but fails with $h_{k}=2.00$, which verifies the effectiveness of Algorithm 1.

Example 2 (Protocol design for undirected graph case using Theorem 4). Since a large scope of industrial applications can be modeled as mass-spring systems, we then consider that a multi-agent system consists of four two-mass-spring systems [32]. The system is shown in Fig. 5. Define the state vector of node $i$ as $x_{i}=\left[x_{i, 1}, x_{i, 2}, x_{i, 3}, x_{i, 4}\right]^{T}=\left[y_{i, 1}, \dot{y}_{i, 1}, y_{i, 2}, \dot{y}_{i, 2}\right]^{T}$. The two-mass-spring system state equation can be written in the form of (2) with

$A=\left[\begin{array}{cccc}0 & 1 & 0 & 0 \\ \frac{-k_{1}-k_{2}}{m_{1}} & 0 & \frac{k_{2}}{m_{1}} & 0 \\ 0 & 0 & 0 & 1 \\ \frac{k_{2}}{m_{2}} & 0 & \frac{-k_{2}}{m_{2}} & 0\end{array}\right], \quad B=\left[\begin{array}{c}0 \\ \frac{1}{m_{1}} \\ 0 \\ 0\end{array}\right]$.

The initial states are given as:

$x_{1}(0)=[1.2589,1.6232,-1.4921,1.6535]^{T}$,
$x_{2}(0)=[0.5294,-1.6098,-0.8860,0.1875]^{T}$,
$x_{3}(0)=[1.8300,1.8596,-1.3695,1.8824]^{T}$,
$x_{4}(0)=[1.8287,-0.0585,1.2011,-1.4325]^{T}$,

and the communication topology $\mathcal{G}$ is described in Fig. 6. In the example, we choose $m_{1}=1.1 \mathrm{~kg}, \quad m_{2}=0.9 \mathrm{~kg}, \quad k_{1}=1.5 \mathrm{~N} / \mathrm{m}$, $k_{2}=1 \mathrm{~N} / \mathrm{m}, Q=0.1 \times I_{4}, R=0.2, h=0.2$. The control task is to design a sub-optimal protocol gain $K^{*}$ so that guaranteed cost consensus is achieved with a sub-minimum guaranteed cost $\gamma^{*}$.

It is assumed in our example that the displacements and velocities of both mass 1 and mass 2 are measurable for all agents. Now we are ready to determine $K^{*}$. By solving SDP-2 for given

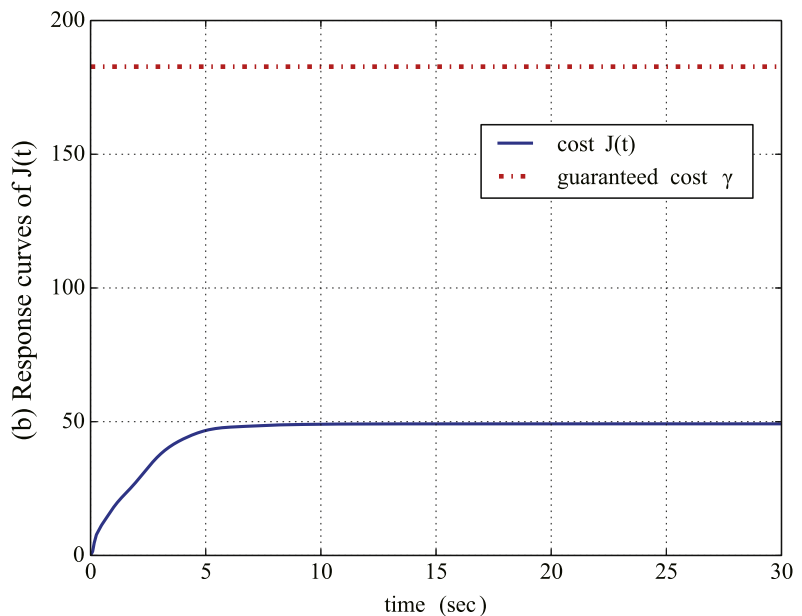

Fig. 7. Consensus process of Example 2 with $h=0.2$. (a) State trajectories and (b) energy consumption. 
$\epsilon_{1}=1, \beta=0.5$, we obtain $\sigma_{2}=1, \sigma_{N}=4$ and

$K^{*}=\left[\begin{array}{llll}-0.4143 & -1.0598 & 0.1734 & -0.5133\end{array}\right], \quad \bar{\gamma}^{*}=182.8$.

The response curves of states and cost are shown in Fig. 7. The consensus is reached, and the cost function value is upper-bounded by $\bar{\gamma}^{*}$, which verifies the effectiveness of Theorem 4 . Note that we can get the same $K^{*}$ and $\bar{\gamma}^{*}$ by solving SDP-1 with the same tuning parameters, but solving SDP-2 use less time (0.7730 s) than that by solving SDP-1 (1.3609 s) on the same computational platform. Moreover, by choosing $\epsilon=0.01$, the allowable sampling interval bound is enlarged to $\bar{h}=0.49$ by Algorithm 1 .

\section{Conclusion}

In this paper, we investigate the guaranteed cost consensus problem for general LTI multi-agent systems with sampled-data information. A sufficient guaranteed cost consensus condition is given in terms of LMIs by applying a refined Lyapunov functional analysis. Reduced-order protocol design methods are proposed, which help a lot in reducing the computation burden while there are massive agents. Based on these theoretic results, LMI-based algorithms are given to find the sub-optimal protocol gain and the maximum allowable sampling interval bound. Note that we construct a novel equivalent system in this paper, which successfully converts guaranteed cost consensus to guaranteed cost stabilization. By utilizing the equivalent relation, extensive control and optimal control results can be extended to consensus process. Future works will focus on extending these results to multi-agent systems.

\section{Acknowledgments}

The authors would like to thank anonymous reviewers for their constructive suggestions and comments which improve substantially the original manuscript. This paper is partly supported by the National Science Foundation of China (61473183, 61521063, U1509211), Program of Shanghai Subject Chief Scientist (14XD1402400).

\section{References}

[1] Fax JA, Murray RM. Information flow and cooperative control of vehicle formations. IEEE Trans Autom Control 2004:49(9):1465-76.

[2] Ogren P, Fiorelli E, Leonard NE. Cooperative control of mobile sensor networks: adaptive gradient climbing in a distributed environment. IEEE Trans Autom Control 2004;49(8):1292-302.

[3] Ren W, Beard RW. Consensus seeking in multiagent systems under dynamically changing interaction topologies. IEEE Trans Autom Control 2005;50 (5):655-61.

[4] Sarlette A, Sepulchre R, Leonard NE. Autonomous rigid body attitude synchronization. Automatica 2009;45(2):572-7.

[5] Jadbabaie A, Lin J, Morse A. Coordination of groups of mobile autonomous agents using nearest neighbor rules. IEEE Trans Autom Control 2003:48 (6):988-1001.
[6] Olfati-Saber R, Murray RM. Consensus problems in networks of agents with switching topology and time-delays. IEEE Trans Autom Control 2004;49 (9):1520-33.

[7] Hong Y, Hu J, Gao L. Tracking control for multi-agent consensus with an active eader and variable topology. Automatica 2006;42(7):1177-82.

[8] Yu W, Chen G, Cao M. Some necessary and sufficient conditions for secondorder consensus in multi-agent dynamical systems. Automatica 2010;46 (6):1089-95.

[9] Li Z, Duan Z, Chen G, Huang L. Consensus of multiagent systems and synchronization of complex networks: a unified viewpoint. IEEE Trans Circuits Syst I: Reg Pap 2010;57(1):213-24.

[10] Zhang H, Lewis FL, Das A. Optimal design for synchronization of cooperative systems: state feedback, observer and output feedback. IEEE Trans Autom Control 2011;56(8):1948-52.

[11] Wen G, Duan Z, Yu W, Chen G. Consensus of multi-agent systems with nonlinear dynamics and sampled-data information: a delayed-input approach. Int J Robust Nonlinear Control 2013;23(6):602-19.

[12] Li S, Du H, Lin X. Finite-time consensus algorithm for multi-agent systems with double-integrator dynamics. Automatica 2011:47(8):1706-12.

[13] Du H, Li S, Shi P. Robust consensus algorithm for second-order multi-agent systems with external disturbances. Int J Control 2012;85(12):1913-28.

[14] Cao Y, Yu W, Ren W, Chen G. An overview of recent progress in the study of distributed multi-agent coordination. IEEE Trans Ind Informatics 2013;9 (1):427-38.

[15] Gao Y, Wang L, Xie G, Wu B. Consensus of multi-agent systems based on sampled-data control. Int J Control 2009;82(12):2193-205.

[16] Cao Y, Ren W. Multi-vehicle coordination for double-integrator dynamics under fixed undirected/directed interaction in a sampled-data setting. Int J Robust Nonlinear Control 2010:20(9):987-1000.

[17] Gao Y, Wang L. Sampled-data based consensus of continuous-time multiagent systems with time-varying topology. IEEE Trans Autom Control 2011;56 (5):1226-31.

[18] Liu H, Xie G, Wang L. Necessary and sufficient conditions for solving consensus problems of double-integrator dynamics via sampled control. Int J Robust Nonlinear Control 2010;20(15):1706-22.

[19] Ma Q, Xu S, Lewis FL. Second-order consensus for directed multi-agent systems with sampled data. Int J Robust Nonlinear Control 2014;24(16):2560-73.

[20] Guo G, Ding L, Han QL. A distributed event-triggered transmission strategy for sampled-data consensus of multi-agent systems. Automatica 2014;50 (5):1489-96.

[21] Huang N, Duan Z, Chen GR. Some necessary and sufficient conditions for consensus of second-order multi-agent systems with sampled position data. Automatica 2016;63:148-55.

[22] Wang Z, Xi J, Yao Z, Liu G. Guaranteed cost consensus for multi-agent systems with switching topologies. Int J Robust Nonlinear Control 2015;25(16):3099-112.

[23] Xi J, Yu Y, Liu G, Zhong Y. Guaranteed-cost consensus for singular multiagent systems with switching topologies. IEEE Trans Circuits Syst I: Reg Pap 2014;61(5):1531-42.

[24] Wang Z, Xi J, Yao Z, Liu G. Guaranteed cost consensus problems for secondorder multi-agent systems. IET Control Theory Appl 2015;9(3):367-73.

[25] Zhao Y, Guo G, Ding L. Guaranteed cost control of mobile sensor networks with Markov switching topologies. ISA Trans 2015;58:206-13.

[26] Fridman E. A refined input delay approach to sampled-data control. Automatica 2010;46(2):421-7.

[27] Li Z, Duan Z. Cooperative control of multi-agent systems: a consensus region approach. CRC Press, Boca Raton, FL; 2014

[28] Cao Y, Ren W. LQR-based optimal linear consensus algorithms. In: 2009 American control conference; 2009. p. 5204-9.

[29] Lewis FL, Vrabie DL, Syrmos VL, Optimal control. 3rd ed., Hoboken, NJ, USA: Wiley; 2012

[30] Fridman E, Shaked U. New bounded real lemma representations for time delay systems and their applications. IEEE Trans Autom Control 2001;46 (12):1973-9.

[31] Lofberg J. YALMIP: a toolbox for modeling and optimization in MATLAB. In: 2004 IEEE international symposium on computer aided control systems design; 2004. p. 284-9.

[32] Lewis FL, Zhang H, Hengster-Movric K, Das A, Cooperative control of multiagent systems: optimal and adaptive design approaches. London, UK: Springer-Verlag, 2014. 